\title{
Improving the Language Mastery through Responsive Environments
}

\author{
A. Vaquero, F. Sáenz, A. Barco* \\ Departamento de Sistemas Informáticos y Programación, Universidad Complutense de \\ Madrid, E-28040 Madrid, Spain \\ * Computing Consultant, E-28040 Madrid, Spain
}

Key words: Computer Based Learning, Constructionism, Language Mastery, Electronic Dictionaries, Lexical Data Base, Ontology.

Abstract: This paper is devoted to a way of intending to improve the language mastery of students in every subject and in a broad range of education levels by using computer-based tools involving linguistic resources. Lexicon concepts useful for language learning are involved in dictionaries and other kinds of linguistic resources. These concepts (vocabulary, meanings, semantic categories, semantic relationships, and taxonomy) are pointed out. Claim is made for using new environments and computer-human interfaces based on these concepts which define the pedagogical goals. Envisioning the foreseen collaborative instruction tasks, claim is made for the constructionist model of learning. Authoring and consulting electronic linguistic resources are the main tasks to reach the defined goals. The conceptual model appropriate for developing any software system taking into account all these principles is discussed, and the reached entity-relationship model is presented. We further present the developed tools for creating bilingual linguistic resources, which could allow to reach the foreseen learning goals. The authoring tool supports consistency of the intended semantics of the lexicon and enables to detect omissions and inconsistencies, and the user tool is a graphical user interface for queries. These tools can be advantageously used in language teaching.

Computers should improve learning and teaching into the classroom Shneiderman, 1995 [1] 


\section{INTRODUCTION}

The challenge of integrating computers in education is mandatory but the traditional resistance of the Education World to innovation is well known. This is the general source of difficulties for exploiting the potentialities of New Technologies into Education [Cornu,1994]. Some very common facts observed in educational centers illustrate this assertion: lack of hardware in schools, lack of quality software, limitations of imported software, lack of reliable and trustworthy reviews or evaluations, etc.

$\%$ Teacher control of computer aided learning

The use of computers in the school must be controlled by the teacher [Cuban,1987] and so, he must be prepared for and aided in assuming this control. Besides the general Computer Literacy that every teacher must currently have, our "user teacher" should have a specific training in "Computers and Education" [Erickson,1994]. Particularly important is the aspect of Language, which we mention here because of its influence in the involved computer-based applications, not only in the direct communication between teacher and learner.

\section{$\%$ Problems of the teacher}

But generally, there is lack of support and appropriate training for teachers. Besides these difficulties, when teachers are committed to apply computers to the classroom, they detect a diversity of problems inhibiting the use of computers across the curriculum [Hodgson,1994], some common ones are: quality of software, access to software, picking out useful software for their own teaching, much of the existing software is difficult to integrate into teaching, often teachers must put in more preparation time before the computer can be used in the classroom, etc. Moreover, as the learning approach changes, new problems appear. The changes are centered in the dynamics of learning, that is, the learner's processes for understanding. This new vision of the nature of learning is based on cognitive theories [Ausubel,1968] [Posner,1989] and it induces new ways of teaching [Tobin,1993]. Acquiring knowledge is an active construction process of the learner.

\section{$\%$ Needs}

With this new vision in mind, the materials needed for teaching could be constructed thinking firstly in the learner more than in the curriculum [Karat,1997]. As a consequence, new computer-based materials and tools should be created for aiding to the learner. Computer-based tools are necessary for off-load heavy, time consuming and boring activities: calculators, symbolic manipulators, graphic and statistical tools, computerbased laboratories, word processors, spread sheets, data bases, graphic 
editors, and so on. More important, the activities involved must induce motivation [Cuban,1997].

$\%$ Student lacks: Language

When weak domains in the student skills and knowledge are detected, it is mandatory to fill the gap by applying appropriate computerbased environments. A specially weak domain is Language. There exist a worrying lack in the mother tongue mastery of the young and not so young people, as it has been recently shown by reliable inquiries made in Spain, but more or less strongly the problem is felt all around the world. The key part of the language misunderstanding is lexicon. There is experimental evidence of reading comprehension dependency of the vocabulary [Johnson, 1978][Thorndike,1973].

$\%$ Solutions

In order to improve the level of language mastery, every pupil ought to handle specific tools with facilities for creation, consulting and modification of language parts. The conventional dictionary, glossary, thesaurus, encyclopedia, etc., electronic (mere electronic copy of the printed ones) or not, have been put in classroom for browsing and consulting purposes. It seems that electronic resources of this kind could motivate to the student more than paper ones. Firstly allowing to the student more easily and quickly to look up terms. Moreover, the computer could allow the development of new tasks with clear pedagogical goals according to the learning model based on constructionism [Cabrera,1995].This implies a new approach to the concept of electronic linguistic resources other than the electronic counterparts of the printed ones [Wilks,1990]. It is necessary to distinguish between machine-readable and machine-tractable dictionaries [Wilks, 1996], as well as for every language resource.

The global pedagogical goal to be reached is word meaning [Quillian,1967]. Word definition is a task for intending to reach the learning of word meaning dependency on other words, as word classification is similar with respect to semantic categories. Specific goals are diverse relationships among terms such as polysemy and synonymy, and their implications into classification. There are involved concepts of the real world and relationships among them such as hypernymy and hyponymy closely attached to ontology [Onishkevich,1992]. The relations between two words of different languages are given through the ontology and its connection to both lexicons. All these goals can be reached following a constructive and collaborative way among students and the teacher in the classroom. This could only be efficiently done with appropriate tools and friendly usable interfaces as a whole responsive environment [Zeltzen,1997].

$\%$ Our proposal 
The intention is to improve the language mastery of students in every subject and in a broad range of education levels by using computerbased tools for authoring and consulting electronic linguistic resources. In order to situate the envisioned tools in their correct instructional place, one must distinguish between constructionist learning in user controlled environments (fully free environments) and navigation in hypermedia ones [Norman,1994].The envisioned tools belong to the first one of these two models of learning, the second one being more appropriate for learning other parts than lexicon [Goldman,1996]. Nonetheless, both are complementary and not absolutely separate [Teusch,1996]. As the second one has been extensively treated in the past [Fernández,1999], the first one is highlighted here. \% Our proposal

In this paper, we present computer-based tools for authoring and consulting multilingual lexical resources supporting ontologies. Our intention is to fill a gap in the niche of constructionist tools for improving the language mastery of students in every subject, which can be applied to a broad range of education levels. Moreover, we believe that each one must build his/her own personal dictionary along his/her entire life. Our tools aid to reach these goals. As far as we know, there are no similar tools to ours described in the literature.

\section{$\%$ Organization}

This paper is organised as follows. In section 2, we highlight some linguistic concepts involved in multilingual dictionaries which are useful for language learning. In section 3, we present the conceptual model of the terminological database incorporated in our tools, which is illustrative for formalising the learning concepts of multilingual dictionaries. Section 4 presents the interface and functionalities of the software tools we have developed. Our first computer-based tool, a user tool for querying a bilingual dictionary, is presented in section 4.1. In section 4.2 we present our second computer-based tool, the author tool for creating bilingual dictionaries, which allows the accomplishment of more learning goals. Finally, section 5 summarises some conclusions and provides hints for future and related work.

\section{LINGUISTIC CONCEPTS INCORPORATED IN OUR PROPOSAL}

Linguistic concepts incorporated in computing systems devoted to Natural Language Processing are pointed out because of their relevance in the definition of the pedagogical goals. These systems subsume the conventional ones (paper dictionaries and the like). The links among these 
concepts and the pedagogical goals are highlighted. Lack of the kind of dictionaries we propose has been felt, as [Fillmore,1992] states:

“... we imagine, for some distant future, an online lexical resource, which we can refer to as a 'frame-based' dictionary, which will be adequate to our aims. In such a dictionary (housed on a workstation with multiple windowing capabilities), individual word senses, relationships among the senses of the polysemous words, and relationships between (senses of) semantically related words will be linked with the cognitive structures (or 'frames'), knowledge of which is presupposed by the concepts encoded by the words."

In addition to the above need, a pedagogical goal is to reach a customisable personal dictionary that allows other functionalities such as personal notes, taxonomies, etc., which are not included in conventional dictionaries.

\subsection{Order, Classification, and Ontology}

$\%$ Order -> classification -> taxonomy

Typically, monolingual dictionaries show an alphabetical order that can be seen as a simple term classification: terms are classified in singletons by its lexicographic form. Other possible less naïve classifications are derivative (root-shape), grammatical, and semantic. Derivative classifications [MaríaMoliner] are not common, and grammatical classifications are not intended for dictionaries. Finally, semantic classification groups terms by semantic categories (for instance, synonym and antonym dictionaries, or ideological dictionaries [Casares].) Semantic categories not also allow meaning classification, but the more meaningful taxonomy of meanings. Conventional lexical databases, such as WordNet [Miller,1995], have term classification such as synonymy (grouped in the so called synsets.) Ontologies go beyond by playing the role of meaning taxonomy [Nirenburg,1995]. Our tools do support this important concept as will be explained along the paper.

\section{$\%$ Order in taxonomy}

Semantic categories are useless for term lookups since meanings will correspond, in general, to a set of (synonym) terms ${ }^{1}$. However, it has an important role on learning by both using and authoring dictionaries because each meaning of a given term (polysemy and/or homonymy) is precisely identified by its semantic category (categories from now on, for the sake of brevity), instead of the usual nonsense sequential

\footnotetext{
${ }^{1}$ Nevertheless, there are other kinds of term lookups as ideological dictionaries show.
} 
number ${ }^{2}$. Therefore, semantic categories provide classification for meanings, and such classification can be arranged in a taxonomy. But this not straightforwardly implies a term order since meanings are abstract ideas that cannot be expressed in general by one distinctive $\operatorname{word}^{3}$. It is commonly acknowledged that the best order for lookups is lexicographic (a derivative classification is a counterexample for this, but it still keeps a lexicographical order by repeating entries and adding links.) Figure 1 resumes the order for taxonomies in a hierarchy; it shows a taxonomy of categories along with the set of terms belonging to each category. From this point of view, there is a complete lexicographic order (provided categories are identified with terms or phrases.) A hierarchy is a natural structure for meaning classification. Each node in the hierarchy corresponds to a category. In principle, every category in the hierarchy can be used, no matter its hierarchy level. It must be noted that every category in the hierarchy contains at least the term which names the category, so that all categories are non-empty. On the other hand, the creation of new categories as intersection of several predefined ones should be avoided, in order to reach compactness.

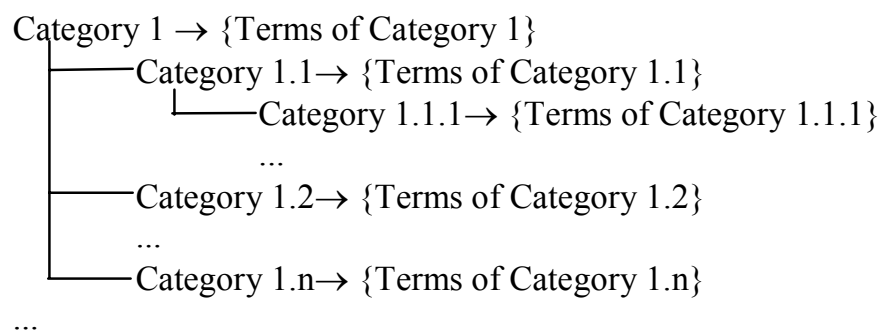

Figure 1. A Taxonomy

\section{EDUCATIONAL GOALS}

From an educational point of view, the goal for students is not to develop a general dictionary (obviously, it is a huge work which linguistic researchers are still carrying out nowadays), but specialised and limited dictionaries that restrict the linguistic domain to make the categorisation of

${ }^{2}$ However, meaning identifications by numbers also show a coarse classification; e.g. Tech. for Technical.

${ }^{3}$ The question is: Which is the best word to represent a meaning? In general, there are several (synonym) words representing the same meaning. 
meanings and the definition of the taxonomy easier. There are a number of advantages in classifying meanings as a taxonomy. First, meaning taxonomy is a useful facility for an electronic dictionary because meanings embody additional semantics which provides more information to the reader (more than that of sequential numbers noted above.) Second, the system may also gain a new dimension because it is possible to automatically generate specialised dictionaries under different categories (a sports dictionary may deal with soccer, tennis, or baseball dictionaries.) Third, it helps to develop a balanced dictionary by adding enough terms from different categories. Having the terms classified, it is easy to check out how many terms are under a given category. Fourth, it also helps to distribute the work between several authors by assigning categories to authors. A team of authors may develop a complete specialised dictionary by dividing the work by categories so that collaborative work is promoted for students. This finally means that the categories must be defined, which implies an added bonus for educational purposes, since it means that students have to organise ideas in a formal way, supported by the implementation of the author tool (covered in the next section.) Additionally, meaning classification can be done under a grammatical criteria, that is, categories can refer to grammatical properties of words (nouns, verbs, ...), so that students can also learn grammatical aspects in this way.

\subsection{Polysemy and Synonymy}

In every language there exists the well-known naming problem [Katzenberg,1993], which consists of two elements: one is polysemy (under the synchronic point of view, that is, embodying polysemy itself and homonymy), by which a term can have several meanings; and the other is synonymy, by which one meaning can be assigned to different terms, as can be observed in Figure 2. In this Figure, Term 1 and Term 2 are synonyms and have a shared meaning, as so for Term 2 and Term3, under another meaning. Moreover, Term 2 is polysemic since it has two possible meanings.

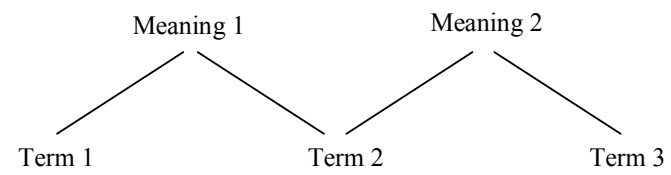

Figure 2. Polysemy and Synonymy 


\subsection{Relationships}

Here we do some remarks about the relationships between categories, meanings and terms. On the one hand, a given term can belong to several categories under different meanings. On the other hand, a given term can belong to several categories under the same meaning. Figure 2.3 shows two categories $(\mathrm{C} 1$ and $\mathrm{C} 2)$ which respectively contain the meanings $\{\mathrm{M} 11$, $\mathrm{M} 12, \mathrm{M}\}$ and $\{\mathrm{M}, \mathrm{M} 21, \mathrm{M} 22\}$. Each meaning has one or more terms associated. The term T2 is associated to meanings M12 and M21, which respectively belong to categories $\mathrm{C} 1$ and $\mathrm{C} 2$. We also show the term $\mathrm{T}$ that is assigned to meaning $\mathrm{M}$, which belongs to both categories $\mathrm{C} 1$ and $\mathrm{C} 2$. Polysemy is present in T2, and synonymy is also present in T3, and T4, as it can be seen. T1 is neither polysemic nor synonym. TC1 and TC2 are the terms used to denote categories $\mathrm{C} 1$ and $\mathrm{C} 2$, respectively.

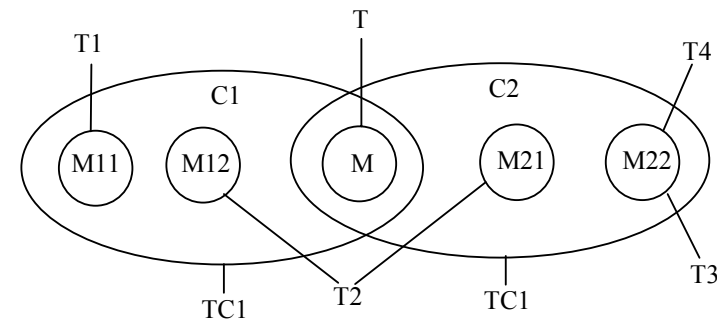

Figure 3. Relationships among categories, meanings and terms. Extensional definition

In this figure, the set of meanings $\{\mathrm{M} 11, \mathrm{M} 12, \mathrm{M}\}$ in $\mathrm{C} 1$ is the extensional definition of category $\mathrm{C} 1$. We must also note that a category has a meaning described by a definition. This figure does not embody this fact. In order to embody the meanings related to categories, we transform the scheme of Figure 3 to the one depicted in Figure 2.4. Now, C1 is the meaning of the category $\mathrm{C} 1$, and $\mathrm{TC} 1$ is the term assigned to such meaning, and the same applies to $\mathrm{C} 2$ and $\mathrm{TC} 2$. Then, we have one more meaning in each category. This meaning is the intensional definition of the category. 


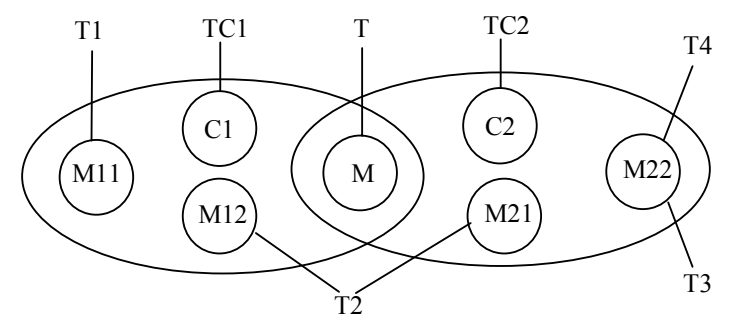

Figure 4. Relationships among categories, meanings and terms. Intensional definition

For a given language, we have a set of terms that holds the relationships with categories and meanings shown in Figure 2.4. If we now think of several languages, the same applies for each one. Then, relationships between terms from different languages come from considering jointly the involved schemes .

\subsection{Foreign Language Learning}

Besides the advantages noted above in using and authoring electronic dictionaries with ontologies for language learning, we also consider foreign language learning under, first, the same lexicon meaning point of view, and second, the relationships between the foreign language and the mother tongue, that is, a multilingual electronic dictionary with support for ontologies. The key idea here is to develop an environment which embodies the linguistic concepts noted above for both the mother tongue and one or more foreign languages. This environment will be a multilingual electronic dictionary with ontologies, and it can allow to reach several teaching goals. First, the assimilation of the foreign lexicon in a constructive way. Second, the mastery of the foreign language when defining meanings in the foreign language. Third, if grammatical criteria is applied to classification, then foreign grammatical properties of the foreign language can be exerted. Finally, the student can comprehend the language independence notion of meanings, so that semantic categories can independently be defined from the language.

\section{CONCEPTUAL MODEL OF THE}

\section{TERMINOLOGICAL DATA B [PSV 1$]$ ASE (TDB)}

There are different TD] - ilt for different purposes. Some of them have incorporated the ontology ture and, so they could possibly be 
used for the pedagogical goals proposed above. But there are a lot of difficulties when intending to do this, not being the less the fact that these very large databases are yet complete or almost complete. So only the tools for building terminological databases are needed. Moreover, the development of this kind of tools must be made taking into account the pedagogical goals which have not been the case of the LDB already built.

Our work in developing the tools is based on a sound conceptual model for the terminological database which shall eventually hold the terms, definitions, meanings, and semantic categories. Since it is intended to deal with two or more languages (bilingual or multilingual dictionaries), we need to represent instances of terms, textual definitions, and textual semantic categories for each language, but, as meanings are not language dependent, we shall use unique representations for them.

The entity-relationship model is used to describe the conceptual model we propose shown in Figure 5. In this Figure (following some recommendations in [Pressman,1997] [Silberschatz,1996]), entity sets are represented with rectangles, attributes with ellipses, and relationship sets with directed and undirected lines. If $\mathrm{B}$ has an incoming line from $\mathrm{A}$, this denotes a one (A) to many (B) mapping cardinality. Double arrows denote many to many mapping cardinalities. Undirected lines denote both one to one mapping cardinalities, when connecting entities, and attributes of entities, when connecting attributes to entities. Relationship set names (not shown in this Figure) label each line.

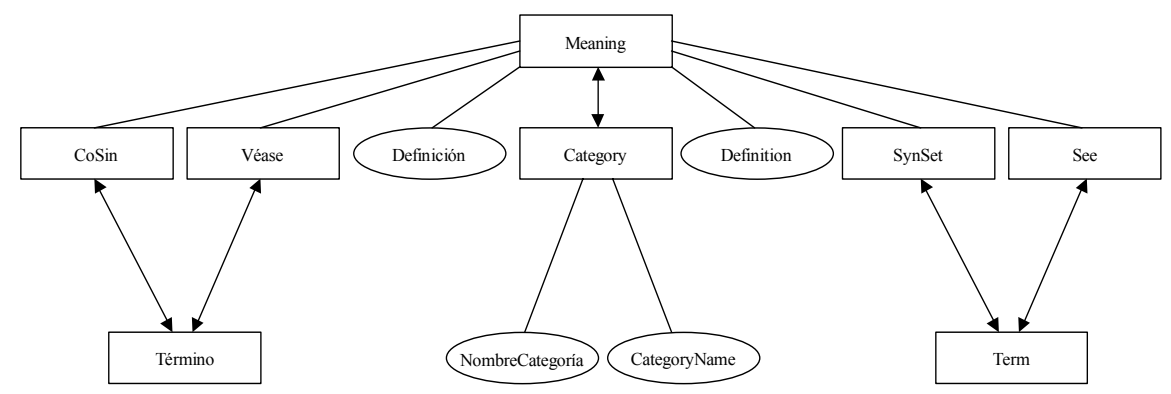

Figure 5. Entity-Relationship Model for an English-Spanish TDB

For the sake of clarity and conciseness, in this Figure we show an instance of a multilingual terminological data base for only Spanish and English languages, but it naturally derives from the general model depicted in Figure 7 (where Li denotes the $i$-th language, $i \in\{1, ., N\}$.) In Figure 5 we depict the entity Meaning, the central entity other entities rest on. In fact, this is the entity which is language independent. The entity SynSet denotes the 
English synonym set (SynSet - Synonym Set.) The relationship set between both entities is one to one. The entity Term represents all the English terms that compose the terminological data base. The relationship set between SynSet and Term is many to many since a synonym set contains several terms, and a term may be contained in several synonym sets (obviously, with different meanings.) Figure 6 embodies this idea, in which Term 1 and Term 2 are synonyms and has a shared meaning, as so for Term 2 and Term 3, under another meaning. Moreover, Term 2 is polysemic.

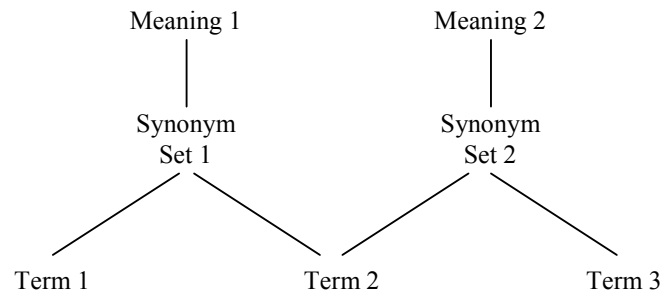

Figure 6. Polysemy and Synonymy related with the synonym sets

The entity See denotes the set of English terms related under a given meaning. The relationship set between Meaning and See is one to one. The relationship set between See and Term is many to many, because a meaning may refer to several English terms, and one term may be polysemic. The entity Category denotes the category each meaning belongs to. The relationship set between Category and Meaning is many to many since many meanings are in a category, and a meaning could be in several categories (this situation is expected to be reduced to the minimum since the goal is to keep the classification as disjoint as possible). This relationship set embodies the fact that our classification is not lexical (there is not a direct relationship between Category and Term) but semantic (we relate meanings to categories, i.e., we categorise meanings.) The entity Category has two attributes: CategoryName and NombreCategoría, which correspond to the textual name of the category in each considered language, English and Spanish, respectively. Meaning has two attributes: Definition and Definición, which correspond to the textual definition in the same considered languages. The remaining entities (CoSin, Véase, Término) are homologous to the respective entities (SynSet, See, Term.) 


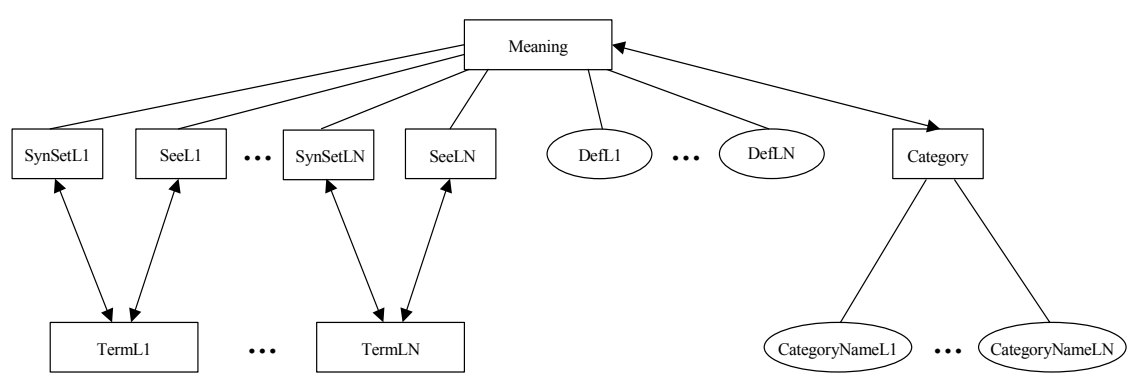

Figure 7. Entity-Relationship Model for a Multilingual TDB

The logical and physical models for the development of any terminological data base following the principles above expressed have to be based on this conceptual model.

\section{INTERFACE AND FUNCTIONALITIES OF}

\section{THE TOOLS}

This section briefly describes the interfaces and functionalities of the user tool and the author tool we have developed.

\subsection{The User Tool}

We have developed an user tool, a query interface which allows us to easily recover the information about both English and Spanish terms as well as their relationships from the so-called terminological data base. This database holds the terms, categories, their attributes, and the relationships. The interface allows the user to navigate the semantic categories, also allowing to retrieve the relevant information of any term (definition, other related terms, translation, synonyms, ...)

The Start window of this tool allows the user to select the base language (i.e., the source language for translations and for representing ::dialogues) among the available languages by pressing its button (from now on, we consider a bilingual dictionary so that it is unnecessary to select the source language or the target language.)

This action pops up the Semantic Category window, as shown in Figure 8; its left pane shows the semantic categories structured as a tree, and the right pane, all the words under the highlighted semantic category. The total number of terms is showed on top of the right pane. The nodes in 
the tree can be clicked in order to expand or contract semantic categories subtrees. A text box is used for term lookups so that the closest word to the substring typed is shown in the right pane. Pressing Enter or double-clicking the highlighted word yields to the Query window. This window shows the relevant information about the selected term: its definition, comments, the list of semantic categories it belongs to (the one corresponding to the shown definition is highlighted), the synonym set and the list of related terms. It also displays a navigation history. It is possible to select another semantic category in this window, which results in updating all the relevant information. Direct access to the terms in both the synonym and related terms windows is allowed by double-clicking.

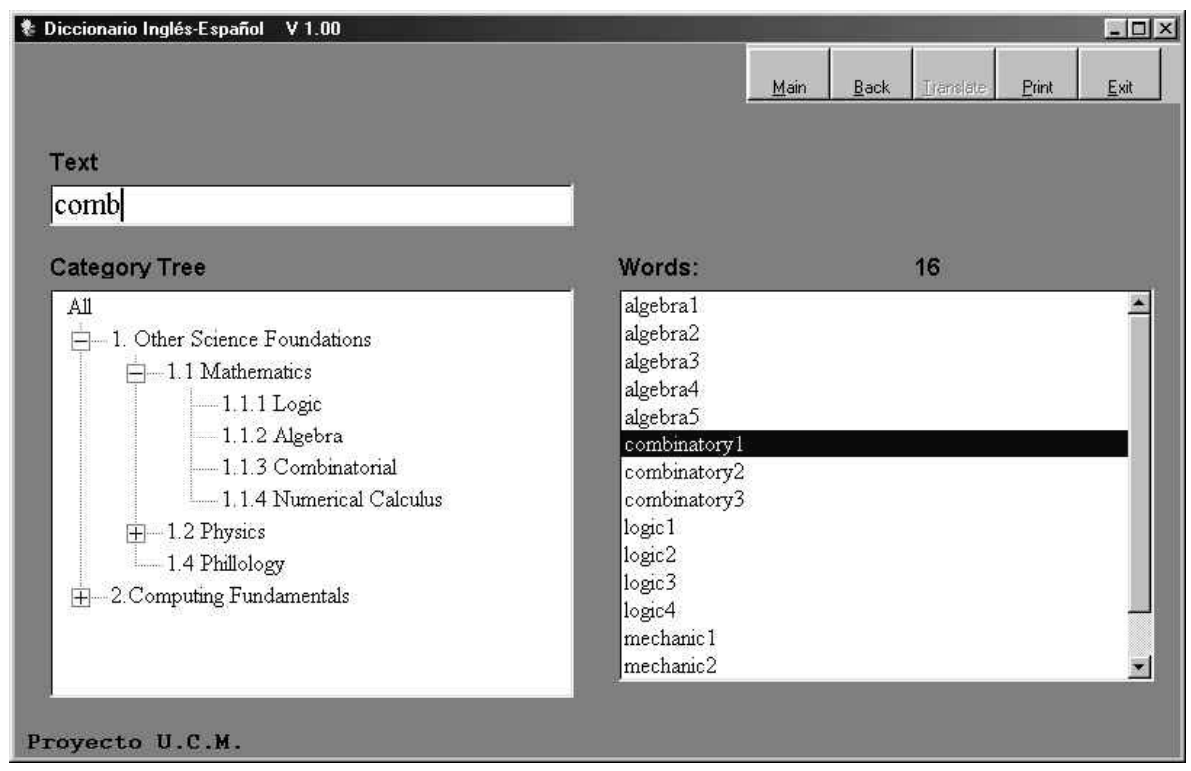

Figure 8. Semantic Category Window

The Semantic Category window has a control box with buttons to activate the return to the Start window, navigate backwards, translate the selected word, print, and exit the interface. The Translate button offers one of the main functionalities of this interface, i.e., the translation from the (source) base language to the target language and, when pushed, it pops up the Translation window (Figure 9). This window shows a first field for the term in the first language, and a second field for the term in the second language. There are also navigation buttons for searching other terms in the same semantic category under an alphabetical order. It is possible to translate from the first or from the second language by using two buttons which express the two possible translation directions. Also, the Go to buttons 
allow us to go to the Semantic Category window for the selected term. This completes the overall description of the functionalities of the user tool.

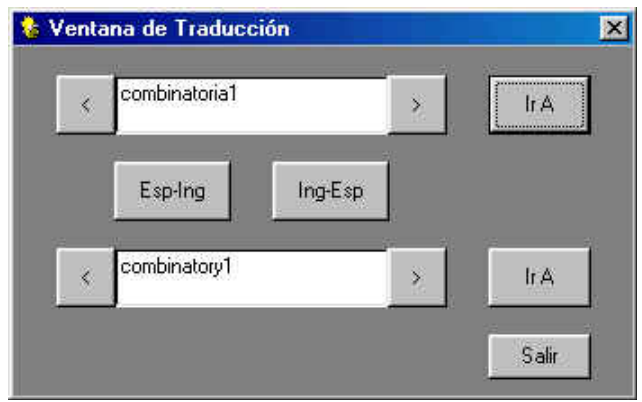

Figure 9. Translation Window

\subsection{The Author Tool}

The author tool allows the author to add new terms to the terminological data base, and all the relevant information, such as its definition, semantic categories, meanings, synonym sets, and related terms. We have developed a Spanish user interface for this tool (easily rewritable to other language), and it consists mainly of one Author window, as shown in Figure 10. It has several management areas (indicated by superimposition in this figure) which are explained next. 


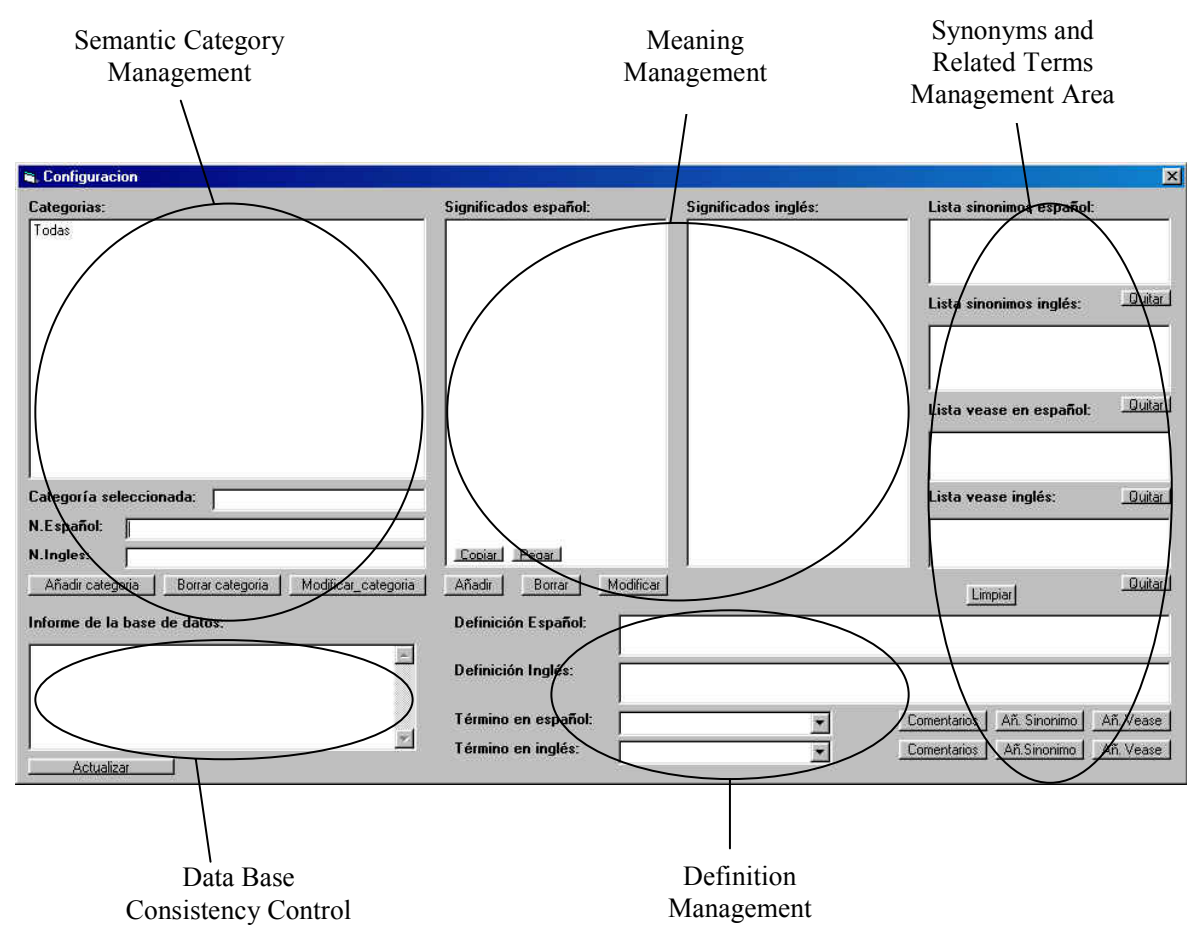

Figure 10. Author Window

\subsubsection{Semantic Category Management}

This area is intended for managing all the operations related to semantic categories, as illustrated in Figure 11 with a fragment of a taxonomy. It has several controls: a hierarchical view of the semantic categories (with expand/collapse functionality), text fields for the semantic category names (English and Spanish), and the buttons Add Category, Delete Category, and Modify Category. The insertion point when adding a new semantic category is the highlighted semantic category, and the Spanish and English texts for the semantic category name must be typed in the aforementioned text fields. 


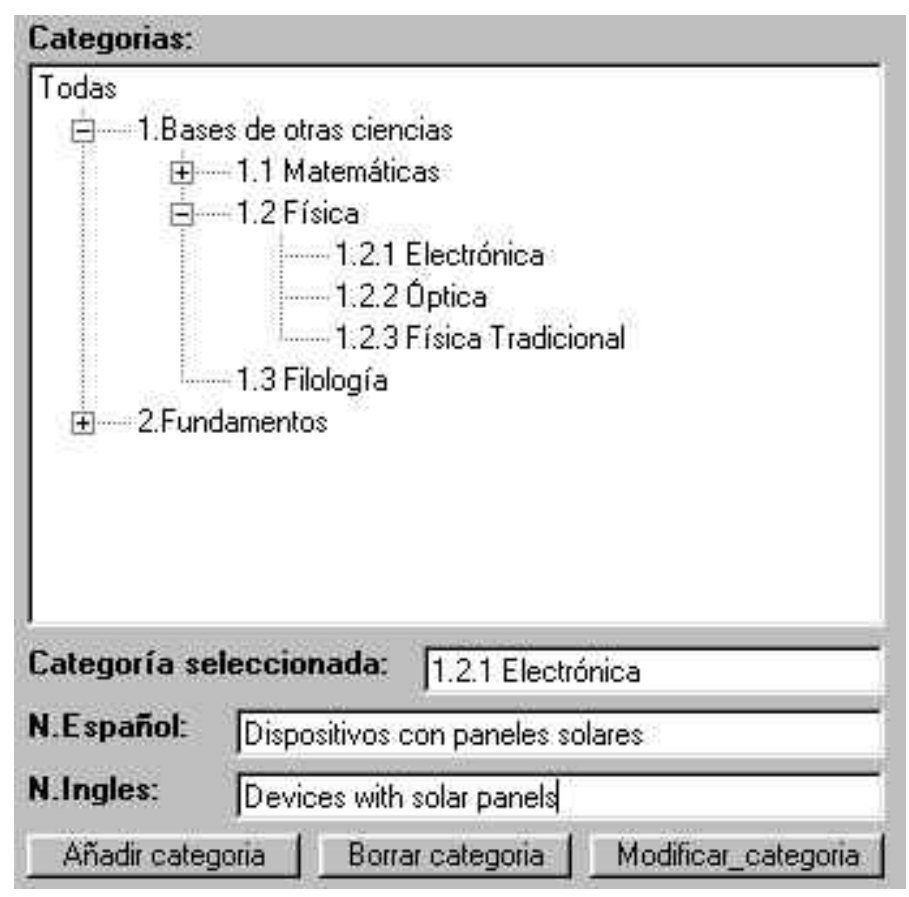

Figure 11. Semantic Category Management Area

\subsubsection{Meaning Management}

The area for meaning management, illustrated in Figure 12, consists of two lists for the meanings in both languages and the buttons Add, Delete, and Modify for addition, deletion, and modification of meanings, as well as buttons for edition (Copy and Paste buttons.) These lists shows the meanings in the form Term -> Definition for the highlighted category, so that one can see several meanings for the same term. Moreover, when a pair Term $->$ Definition is selected, the corresponding Term $->$ Definition translation is automatically highlighted; there is a one-to-one mapping between meaning representation in all the languages. It should also be noted that meanings, which are language independent, are shown with the best representation we have in a given language, i.e., a pair Term -> Definition, since there are no other pair Term $->$ Definition2 with the same meaning (note that is the same term in both pairs.) 


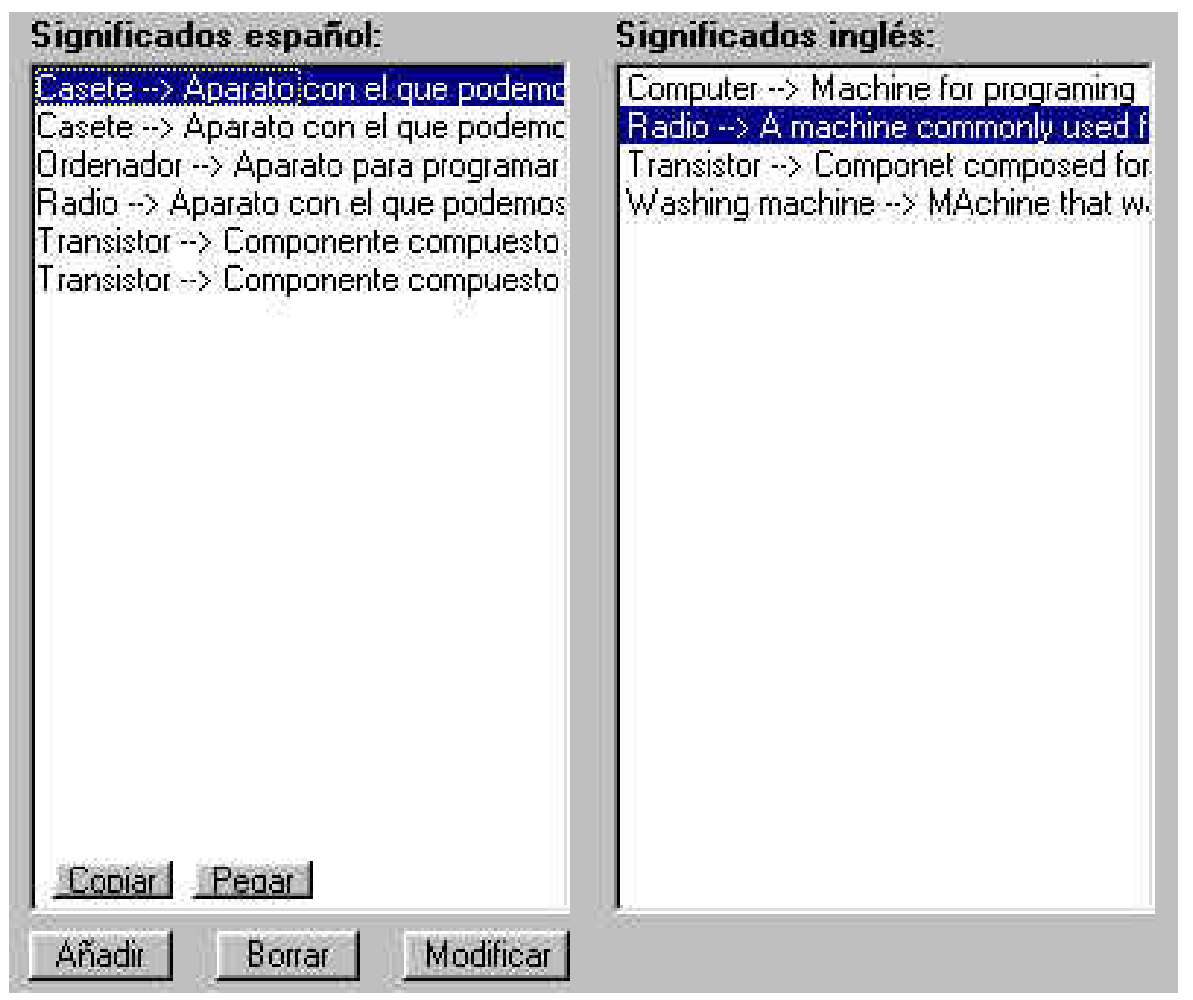

Figure 12. Meaning Management Area

\subsubsection{Synonyms and Related Terms Management Area}

The area on the right in Figure 12 has four lists for the synonyms, and related terms in both languages which correspond to the highlighted meaning in the Meaning Management area.

\subsubsection{Database Control Area}

This area contains the button Update, which is used to modify the database with the typed information, and to obtain a report (text box Data Base Report) about consistency of the database (Figure 3.3). Up to now, consistency detection only detects lack of textual definitions for terms, but it can be extended in order to detect other inconsistencies or omissions. This is quite important when authoring dictionaries, since a dictionary cannot be consistently built at each step, but it is constructively built from terms to relationships between terms (polysemy, synonymy.) For instance, this tool can be extended in order to give hints for detecting circular definitions (there 
are commercial dictionaries with this failure), for detecting possible lacks of synonym and related terms, and so on.

\section{CONCLUSIONS}

To face the challenge of fulfilling the lack of language mastery new tools and interfaces are needed. These tools have to be a consequence of the linguistic concepts involved, the defined pedagogical goals, and the chosen constructionist learning model, as exposed above. So, a sound entityrelationship model which embodies these linguistic concepts has been established, and it is well suited for developing lexical databases with a rich structure. The conceptual model presented here supports the concept of ontology.

\section{$\%$ Hoped Educational Achievements}

We have developed tools for aiding to improve the language learning. These tools are a consequence of the defined pedagogical goals and the linguistic concepts involved, as shown above. Putting to work in service these represents a hope for educational achievement, and the experiences will guide the additions and modifications to improve their efficacy.

\section{$\%$ Future work}

These tools can be enhanced in several ways. To mention only a few, firstly, both the user and author tool can be deployed in a Web context in order to allow centralized information for queries, and, what is more important, to allow collaborative work at a distance. Secondly, they can be extended with phonetic search. And thirdly, the author tool database control can be improved with the identification of not defined words in textual definitions, which can help for completeness.

\section{$\%$ Further applications}

Classification of meanings, as we have emphasized before, is important for two challenging applications. First, to integrate a terminological data base into a multilingual knowledge base. And second, for information retrieval. A sound conceptual model is necessary to integrate a terminological data base into a multilingual lexical one. We have in mind lexical knowledge bases based on ontologies (e.g., MikroKosmos [MikroKosmos]), rather than monolingual on line lexical resources (e.g., WordNet [Miller,1995].) Since the conceptual model we develop here is coherent with the concept of ontology, the implementation of a terminological data base from that conceptual model must facilitate its integration into an ontology based lexical knowledge base. So we can assume the implementations to be built from this model will accomplish the 
conditions to be rightly used as essential steps of information retrieval operations.

\section{REFERENCES}

[Ausubel,1968] D.P. Ausubel, "Educational Psychology: A cognitive View", New York, Holt, Reinhart and Winston, 1968.

[Cabrera,1995] A. Cabrera, "Informática Educativa: La revolución

constructivista”. Informática y Automática, Vol. 28, n. 1, marzo 1995.

[Casares] Ideological Spanish Dictionary.

[Cornu,1994] B. Cornu, "New Technologies:Integration into Education".

Proceedings of the Working Conference", Integrating Information Technology into Education”, IFIP, Barcelona, pp.15-24, October 17-21, 1994.

[Cuban,1987]L. Cuban, "Teachers and Machines: The classroom use of technology since 1920”. Teachers College Press, Columbia University, New York, 1987.

[Cuban,1997]L. Cuban, Foreword in "Teaching with Technology: Creating Student Centered Classrooms". H.J. Sandholtz, C. Rigstaff, and D.C. Dwyer, Eds., Teachers College Press, New York, 1997.

[Erickson,1994] F.J. Erickson, and J.A. Yonk, "Computer Essentials in Education. The teaching tools". McGraw-Hill Book Co., 1994.

[Fernández,1999] A. Fernández-Valmayor, C. López-Alonso, S. Arlette, and B. Fernández-Manjón, "The Design of a Flexible Hypermedia System: Integrating an Interactive Learning Paradigm for Foreign Language Text Comprehension", International Working Conference on Building Electronic Educational Environments, IFIP, Irvine, California, pp. 51-65, 1999.

[Fillmore,1992] C.J. Fillmore, and B.T. Atkins, "Toward a frame-based lexicon: The semantics of RISK and its neighbors”, Lehrer and Kittay, pp. 75-102, 1992.

[Goldman,1996] S.R. Goldman, "Reading,Writing,and Learning in Hipermedia Environments", Cognitive Aspects of Electronic Text Processing (Ed.H.Van Oostendorp and S. Mul), Norwood, NJ. Ablex Publications, 1996.

[Hodgson,1994] B. Hodgson, "The roles and the needs of the teacher". Proceedings of the Working Conference "Integrating Information Technology into Education", IFIP, Barcelona, pp. 25-34, October 17-21, 1994.

[Johnson,1978] D.D. Johnson, and P.D. Pearson, ”Teaching Reading Vocabulary”, Ed. Holt, Reinhard \& Winston, New York, 1978.

[Karat,1997] J. Karat, "Evolving the scope of user-centered design"

Communications of the ACM, Vol. 40, N. 7, July, 1.997.

[MaríaMoliner] Derivative Spanish Dictionary.

[MikroKosmos] http://crl.nmsu.edu/Research/Projects/mikro/index.html

[Miller,1995] G. Miller, "WordNet: A Lexical Data Base for English", Communications of the ACM, Vol. 38, 11, 1995.

[Nirenburg,1995] S. Nirenburg, V. Raskin, and B. Onyshkevich, "Apologiae Ontologiae", Proceedings of the Sixth International Conference on Theoretical and Methodological Issues in Machine Translation, Center for Computational Linguistics, Catholic University, Leuven, Belgium, pp. 106-114, 1995.

[Norman,1994] K. Norman, "Navigating the educational space with HyperCourseware". Hypermedia, Vol. 6, enero 1994.

[Onyshkevych,1992] B. Onyshkevych, and S. Nirenburg, "Lexicon, Ontology, and Text Meaning", Lexical Semantics and Knowledge Representation, J. Pustejovsky, Ed., Springer-Verlag, 1992. 
[Posner,1989] M.I. Posner(Ed.), "Foundations of Cognitive Science".

Cambridge, Mass., MIT press, 1989.

[Pressman,1997] R.S. Pressman, "Software Engineering. A Practitioner's

Approach", McGraw-Hill, 1997.

[Quillian,1967] M. R. Quillian, "Word Concepts: A Theory and Simulation of Some Basic Semantic Capabilities". Brachmen, R. J. y Levesque, H. J., Eds., Reading in Knowledge Representation. Morgan Kaufman, 1.967.

[Shneiderman,1995]B. Shneiderman, M. Alavi, K. Norman, and E.Y.

Borkowski, "Windows of opportunity in electronic classroom", Communications of the ACM, Vol. 38, N. 11, November, 1.995.

[Silberschatz,1996] A. Silberschatz, H.F. Korth, S. Sudarshan, "Data Base System Concepts", WCB/McGraw-Hill, 1996.

[Teusch,1996] P. Teusch, T. Chanier, Y. Chevalier, D. Perrin, F. Mangenot, J.P. Narcy, and J.de Saint Ferjeux, "Environnements interactives pour l'apprentissage en langue étrangère". Hipermedias et Apprentissage, 3 (Ed. E. Brouillard), 1996, pp. 247-256.

[Thorndike,1973] R.L. Thorndike, "Reading Comprehension Education in Fifteen Countries", Ed. Wiley, 1973.

[Tobin,1993] K. Tobin, and D. Tippings, "Constructivism as a referent for teaching and learning". K. Tobin (Ed.), "The practice of Constructivism in Science Education”. AAAS Press. Washington, DC, 1993, pp. 3-21.

[Wilks, 1990] Y.A. Wilks, D.C. Fass, C.M. Guo, J.E. McDonald, T. Plate, and B.M.Slator, "Providing machine tractable dictionary tools". Machine Translation, 5, 1990, pp. 99-151.

[Zeltzen,1997] D. Zeltzen and R. K. Addison, "Responsive virtual environments", Communications of the ACM, Vol. 40. N. 8, August, 1997. 